Discussion Paper No. 09-075

\title{
Ökonomische Aspekte der privaten Durchsetzung des Kartellrechts
}

Patrick F.E. Beschorner und Kai Hüschelrath

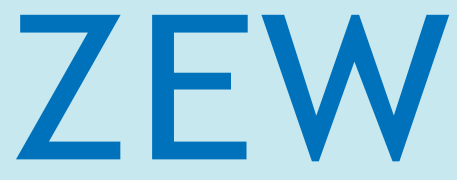

Zentrum für Europäische Wirtschaftsforschung $\mathrm{GmbH}$

Centre for European

Economic Research 
Discussion Paper No. 09-075

\title{
Ökonomische Aspekte der privaten Durchsetzung des Kartellrechts
}

\author{
Patrick F.E. Beschorner und Kai Hüschelrath
}

Download this ZEW Discussion Paper from our ftp server:

ftp://ftp.zew.de/pub/zew-docs/dp/dp09075.pdf

Die Discussion Papers dienen einer möglichst schnellen Verbreitung von neueren Forschungsarbeiten des ZEW. Die Beiträge liegen in alleiniger Verantwortung der Autoren und stellen nicht notwendigerweise die Meinung des ZEW dar.

Discussion Papers are intended to make results of ZEW research promptly available to other economists in order to encourage discussion and suggestions for revisions. The authors are solely responsible for the contents which do not necessarily represent the opinion of the ZEW. 


\section{Das Wichtigste in Kürze}

Bei Verstößen gegen geltendes Wettbewerbsrecht entstehen typischerweise Schäden durch überhöhte Preise und entgangenen Gewinn bei nachgelagerten Wirtschaftsstufen. Hierfür besteht ein gesetzlicher Schadensersatzanspruch. Auf europäischer Ebene wird derzeit ein Rechtsrahmen diskutiert, mit dem eine einheitliche Behandlung in den Mitgliedsstaaten gewährleistet werden soll. Im Rahmen dieser Arbeit wird aufgezeigt, welche Anreize entstehen und daher bei der Ausgestaltung der einschlägigen Regelungen $\mathrm{zu}$ berücksichtigen sind, um die bisherigen Mechanismen zur Abschreckung neuer Kartelle und Aufdeckung bestehender Kartelle nicht zu beschädigen.

Wir stellen fest, dass die private Durchsetzung von Schadensersatzansprüchen einen Beitrag leistet, um Kartelle zu verhindern und die Schäden aufgedeckter Kartelle zu kompensieren. Die präventive Wirkung ergänzt die bisherigen Instrumente zur Aufdeckung von Kartellen. Daher werden Hardcore-Kartelle, die relativ leicht von potentiell Geschädigten aufgedeckt werden können, mit geringerer Wahrscheinlichkeit überhaupt erst eingerichtet.

Nach Aufdecken eines Kartells sollen die Betroffenen ihren Schaden in vollem Umfang, also die Verteilungswirkung und den Allokationseffekt, geltend machen dürfen. Kartellmitglieder sollen die passing-on defense entgegenhalten dürfen. Dies bedeutet, dass ein von überhöhten Preisen betroffenes Unternehmen einen Teil des Preisaufschlages an seine Kunden weitergibt. Diese Weitergabe von überhöhten Preisen muss dieses Unternehmen gegen sich geltend machen lassen. Nur so kann prinzipiell eine Kompensation des vollen Schadens erfolgen. Dies wird nur gehemmt durch Kosten der Rechtsdurchsetzung. Die Gefahr der missbräuchlichen Kollusion zwischen Schädiger und mittelbar Geschädigten bei der Anwendung der passing-on defense besteht nur in besonderen Einzelfällen.

Dem Effekt, dass Kartelle weniger wahrscheinlich werden, steht entgegen, dass die Kronzeugenregelung weniger attraktiv wird, wenn der Kronzeuge sich mit dieser Rolle zugleich selbst Schadensersatzansprüchen aussetzt. Dies kann durch die Ausgestaltung seines Haftungsumfanges begrenzt werden und bedarf weiterer Klärung. Die Attraktivität der Kronzeugenregelung sinkt daher nicht per se. Die Zuordnung des Gesamtschadens auf die einzelnen Kartellmitglieder lässt sich konzeptionell nicht durchführen. Insbesondere ist die Zuordnung des Schadens eines betroffenen Unternehmens zu dem Kartellmitglied, mit dem es in einer Vertragsbeziehung steht, inkorrekt. Ursächlich ist alleine die Kartellvereinbarung, an der alle Kartellmitglieder beteiligt sind.

Insgesamt befürworten wir die Initiative der Europäischen Kommission, einen Rechtsrahmen für die private Durchsetzung des Kartellrechts zu schaffen. 


\title{
Ökonomische Aspekte der privaten Durchsetzung des Kartellrechts
}

\author{
Patrick F.E. Beschorner*, Kai Hüschelrath*
}

\begin{abstract}
Kurzzusammenfassung
Bei Verstößen gegen geltendes Wettbewerbsrecht entstehen typischerweise Schäden durch überhöhte Preise und entgangenen Gewinn bei nachgelagerten Wirtschaftsstufen. Hierfür besteht ein gesetzlicher Schadensersatzanspruch. Auf europäischer Ebene wird derzeit ein Rechtsrahmen diskutiert, mit dem eine einheitliche Behandlung in den Mitgliedsstaaten gewährleistet werden soll. Die Möglichkeit der privaten Durchsetzung des Kartellrechts hat zwei Effekte: Anreize ein Kartell einzugehen und Anreize innerhalb eines bestehenden Kartells. Betroffene Unternehmen haben einen stärkeren Anreiz ein Kartell aufzudecken, dem sie ausgesetzt sind. Dies reduziert die Wahrscheinlichkeit, dass sich Kartelle bilden. Bei bestehenden Kartellen kann die Anwendung der Kronzeugenregelung weniger attraktiv werden, wenn ein Unternehmen zwar ein Bußgeld vermeidet, sich aber Schadensersatzansprüchen aussetzt. Daher ist dies in der Ausgestaltung der Haftungsregelung gegen den Kronzeugen zu berücksichtigen.
\end{abstract}

Keywords: Private Kartellrechtsdurchsetzung, Kartelle, Kronzeugenregelung JEL: $\quad$ K21, K42, L44

* $\quad$ Zentrum für Europäische Wirtschaftsforschung GmbH (ZEW), Postfach 1034 43, 68034 Mannheim, Tel.: +49-(0)-621/1235-175, Fax.: +49-(0)-621/1235-170, E-Mail: beschorner@zew.de

* Zentrum für Europäische Wirtschaftsforschung GmbH (ZEW) und WHU Otto Beisheim School of Management, Burgplatz 2, 56179 Vallendar, E-Mail: hueschelrath@zew.de Diese Arbeit ist mit finanzieller Unterstützung des RNIC Research Network on Innovation and Competition Policy entstanden. Wir danken Florian Bien für seine hilfreiche und ausführliche Kommentierung dieses Manuskripts, sowie den Teilnehmern des 2. Studientages des Studienkreises „Wettbewerb und Innovation“ für die anregende Diskussion. 


\section{A. Einleitung}

Die Möglichkeit der privaten Durchsetzung des Kartellrechts lässt sich im Wesentlichen in zwei Bereiche unterteilen: Einflüsse auf Kartelle vor ihrer Aufdeckung und danach. Zum einen haben Kunden einen Anreiz ein Kartell aufzudecken, wenn sie erwarten, dass darauf hin die Preise sinken, die Qualität sich verbessert oder sonstige Einschränkungen aufgehoben werden. Auf diesen Anreiz hat die Einführung der privaten Durchsetzung von Schadensersatz im Kartellrecht einen stärkenden Einfluss. Zum anderen haben Geschädigte nach Aufdecken eines Kartells ein Interesse Schadensersatz zu erhalten. Der Schwerpunkt der Untersuchung wird im Folgenden auf dem zweiten Bereich liegen.

Mit der Diskussion um die private Kartellrechtsdurchsetzung folgt die EUKommission einer Vorgabe des EuGH. ${ }^{1}$ Damit haben alle Bürger und Unternehmen einen Anspruch auf Schadensersatz gegen diejenigen, die ursächlich aus einer Verletzung des Wettbewerbsrechts einen Schaden herbeiführen. ${ }^{2}$ Die Diskussion im Rahmen des Grünbuchs und des Weißbuchs zielt darauf ab, die rechtlichen Rahmenbedingungen für die Geschädigten zu verbessern, damit sie einen Anspruch auf Schadensersatz wirksamer durchsetzen können. Die hier untersuchten Regelungen beziehen sich auf Wettbewerbsverstöße im Allgemeinen. Dennoch konzentrieren wir uns im Rahmen dieses Aufsatzes auf Kartelle. Diese Beschränkung dient der Klarheit der Argumentation, auch wenn viele Aspekte auch auf andere Arten von Verstößen zutreffen.

Aus ökonomischer Sicht steht der Beitrag der privaten Kartellrechtsdurchsetzung zur Erhöhung der sozialen Wohlfahrt im Vordergrund. ${ }^{3}$ Grundsätzlich sollte ein Akteur die Kosten, die er verursacht, auch selbst tragen. Diese Kosten können sowohl auf der gleichen als auch auf vor- und nachgelagerten Wirtschaftsstufen entstehen. Wenn ein Kartell beispielsweise überhöhte Preise verlangt, sollte es den Schaden seiner Käufer oder Kunden übernehmen. Die Überlegungen zur Effizienz der Rechtsdurchsetzung umfassen aber auch ein Abwägen dieses Prinzips mit den Kosten der Durchsetzung von Schadensersatzansprüchen. Dies spielt beispielsweise bei der Festlegung von Anforderungen an die von Geschädigten vorzubringenden Beweise eine Rolle. In diesem Zusammenhang ist auch die Gefahr eines übermäßigen - möglicherweise missbräuchlichen - Gebrauchs dieses Instruments zu nennen.

Um auf diese verschiedenen Aspekte eingehen zu können, gliedert sich dieser Aufsatz zunächst in einen Abschnitt zur Aufdeckung und Verhinderung von Kartellen. Die anschließenden Abschnitte beziehen sich auf die Durchsetzung von Schadensersatzansprüchen nachdem ein Kartell aufgedeckt und festgestellt wurde

\footnotetext{
${ }^{1}$ Rechtssache EuGH v. 20.9.2001, Rs. C-453/99 - Courage/Crehan, Slg. 2001, I-6297.

${ }^{2}$ Siehe Weißbuch, Europäische Kommission (2008b), S.2.

${ }^{3}$ Rubinfeld (2009).
} 
sowie auf mögliche Konflikte mit der bestehenden Kronzeugenregelung. Wir schließen mit einer kurzen Zusammenfassung und einem Ausblick.

B. Veränderte Anreize durch private Kartellrechtsdurchsetzung

\section{Aufdecken und Verhindern von Kartellabsprachen}

Die Stärkung der privaten Durchsetzung von Kartellrecht beeinflusst die Anreize zum Aufdecken von Kartellen ebenso wie die Anreize sie überhaupt einzugehen. ${ }^{4}$ Dass die Möglichkeit der privaten Durchsetzung des Wettbewerbsrechts einen Beitrag dazu leistet, Wettbewerbsverstöße überhaupt aufzudecken, wird in den Dokumenten der EU, dem Grünbuch, dem Weißbuch und den Begleitpapieren nicht thematisiert. Wettbewerbsbehörden arbeiten ex officio an der Aufdeckung von Kartellen. Aufgrund der Kronzeugenregelung können Kartellmitglieder einen Anreiz haben, ein Kartell aufzudecken. Doch auch Geschädigte eines Kartells haben einen Anreiz Wettbewebsverstöße, unter denen sie leiden, aufzudecken.

Typische Geschädigte von Kartellen sind die Unternehmen oder Personen, die Waren oder Dienstleistungen von Kartellmitgliedern beziehen. Ohne den Anspruch auf Schadenersatz besteht für diese Unternehmen oder Personen der Anreiz, die Fortsetzung des Kartells zu unterbinden, allein darin, nicht weiter den negativen Folgen des Kartells ausgesetzt zu sein. Typischerweise sind dies überhöhte Preise.

Mit einer Stärkung der privaten Durchsetzung des Wettbewerbsrechts, insbesondere des Anspruchs auf Schadensersatz, steigert sich dieser Anreiz. Dies bedeutet, dass Geschädigte von sich aus einen höheren Anreiz haben, einen Verstoß zu verfolgen und zur Anzeige zu bringen. Dieser Aspekt sollte nicht vernachlässigt werden, denn gerade die Kunden von Kartellmitgliedern sind mit den Marktgegebenheiten vertraut und können gegenüber Mitarbeitern von Behörden einen entscheidenden Wissensvorsprung haben.

Die Schadensersatzverpflichtung von Kartellmitgliedern hat eine abschreckende Wirkung ebenso wie ein Bußgeld, sollte aber nicht als Instrument zur Abschreckung oder Bestrafung eingesetzt werden. Die Stärke der Wirkung hängt alleine von der Höhe der Zahlung ab, denn die Abschreckung wird durch die Höhe der Summe aller Zahlungen im Falle der Aufdeckung bestimmt. Inwiefern Schadensersatzzahlungen den tatsächlichen Schaden überschreiten können sollen, ist eine Frage der nationalen Wettbewerbspolitik und kann sich zwischen verschiedenen Jurisdiktionen unterscheiden. ${ }^{5,6}$ An dieser Stelle hat die Wettbewerbspolitik zwei Instrumente, mit denen sie unabhängig voneinander die Abschreckung von Kartellen und die

\footnotetext{
${ }^{4}$ Siehe Renda et al. (2007), S.70ff.

${ }^{5}$ Siehe Rubinfeld (2009), S.899ff.

${ }^{6}$ Treble Damages, die ein Dreifaches des tatsächlichen Schadens betragen, werden in den USA unter bestimmten Voraussetzungen zugesprochen.
} 
Anreizwirkung bei den Geschädigten beeinflussen kann. Daher ist es nicht erforderlich die Höhe des Schadensersatzes als Instrument zur Abschreckung zu nutzen, wenn hierfür das Instrument des Bußgeldes zur Verfügung steht.

Ein Schadensersatz, der über den erlittenen Schaden hinausgeht, würde außerdem die Anreize zu einem missbräuchlichen Einsatz von Schadensersatzklagen verstärken. Durch eine unberechtigte oder eine mit geringer Wahrscheinlichkeit berechtigte Schadensersatzklage können der beklagten Partei hohe Kosten aufgebürdet werden. Die Bereitstellung der zur Beweisführung notwendigen Unterlagen verursacht hohe Personalkosten seitens der Kartellanten. Obwohl eine Schadensersatzklage unberechtigt ist, kann die beklagte Partei bereit sein eine Zahlung im Rahmen eines Vergleichs zu leisten, alleine um die noch höheren Kosten einese Gerichtsverfahrens zu vermeiden. Solche in quasi erpresserischer Absicht eingereichten Klagen würden verstärkt auftreten, wenn der Schadensersatz um eine Betrafungskomponente erhöht würde.

Auch wenn Schadensersatz nicht als Strafe mit einer abschreckenden Wirkung gedacht ist, so hat er diese Wirkung. ${ }^{7}$ Wäre es als Strafe mit Abschreckungswirkung gedacht, dann wäre das ideale Strafmaß so hoch, dass die erwartete Sanktion gerade dem Gewinn aus der rechtswidrigen Handlung entspricht. Dann würde sich ein Unternehmen durch seine Beteiligung an einem Kartell nicht besser stellen. Da die Wahrscheinlichkeit, dass die Sanktion verhängt wird, also von einem Betroffenen eingefordert wird, geringer als eins ist, muss die Sanktion höher sein als der tatsächliche Schaden. In Deutschland ist aber ein Anspruch auf Schadensersatz bis auf wenige zu vernachlässigenden Ausnahmen niemals höher als der tatsächliche Schaden. Eine weitere Schwierigkeit wäre, dass man die Wahrscheinlichkeit der Sanktion und der folgenden Schadensersatzforderung ermitteln müsste. Dies ist aber nicht möglich, weil man stets nur Bußgelder gegen aufgedeckte Kartelle verhängen kann und die Wahrscheinlichkeit, dass ein Kartell unentdeckt bleibt sich nur sehr schwer und mit hoher Unsicherheit abschätzen lässt.

\section{Schadenersatz}

Schadenersatzforderungen bei Wettbewerbsverstößen können oft daran scheitern, dass die vermeintlich Geschädigten keinen Verstoß nachweisen können. Dies sei eines der größten Hindernisse bei der privaten Durchsetzung von Schadensersatzansprüchen im Wettbewerbsrecht. ${ }^{8}$ Tatsächlich sind manche Verstöße nur schwer nachzuweisen, insbesondere, wenn nachgewiesen werden muss, dass eine benachteiligende Wirkung gerade das Motiv einer Entscheidung oder eines Verhaltens und nicht nur der Nebeneffekt einer ansonsten legitimen Unternehmensstrategie ist. Der Nachweis des Missbrauchs einer marktbeherrschenden Stellung ist schwer und nur mit relativ hohen Kosten zu

\footnotetext{
${ }^{7}$ Rubinfeld (2009), S.899.

${ }^{8}$ Rubinfeld, (2009), S.895. Europäische Kommission (2008a), S.2.
} 
erbringen. Dagegen ist ein Hardcore-Kartell eindeutig aufgedeckt, wenn die Dokumentation zu illegalen Absprachen einmal vorliegt. Ist ein Verstoß festgestellt worden, gehen das GWB in $\S 33$ IV und das Weißbuch davon aus, dass dem Geschädigten keine Beweispflicht hinsichtlich des Verstoßes mehr obliegt. Dies ist jedoch umstritten. Dabei ist es unerheblich, ob der Geschädigte diesen Nachweis selbst erbracht hat oder anderweitig erbracht wurde.

Die Diskussion um Schadensersatz wirft zwei Fragen auf: Wer soll einen Anspruch auf Schadensersatz erhalten? Und wie wird der Schaden bemessen? Beiden Fragen gehen wir in den folgenden Abschnitten nach. Wegen der Spezifität der passing-on defense, wird dieser Komplex in einem eigenen Abschnitt behandelt.

In welcher Höhe entsteht ein Schaden? Bei der Feststellung des Schadens und der Ursache stellen sich zahlreiche Fragen konzeptioneller Art. Ob schon die Kartellabsprache oder erst die Verweigerung unter dem Kartellpreis an einen Kunden zu verkaufen die Ursache eines möglichen Schadens darstellt, kann strittig sein. Schließlich bedeutet der Vorwurf überhöhter Preise, dass es einen Referenzpreis geben muss, in Vergleich zu welchem der Kartellpreis als zu hoch angesehen wird. Intuitiv ist der Referenzpreis derjenige, der sich ohne die Kartellabsprache eingestellt hätte. Der Schaden des Kunden summiert sich dann aus der Preisdifferenz für jede gekaufte Einheit zuzüglich dem Einkommensverlust aus dem Rückgang der Verkaufsmenge infolge der Kostenerhöhung. Diese beiden Effekte, den Verteilungseffekt und den Allokationeffekt, beschreibt Hellwig (2006) en Détail. Der Schaden besteht also aus zwei Komponenten: Exzessive Kosten und entgangener Gewinn.

Ohne weitere Information kann man nur sagen, dass der Referenzpreis zwischen einem Wettbewerbspreis in Höhe der Grenzkosten der Produktion und dem Monopolpreis liegen wird. Dabei ist es sinnvoll anzunehmen, dass die Marktstruktur ohne die Kartellabsprache identisch gewesen wäre, obwohl es beispielsweise denkbar wäre, dass ohne das Kartell eines oder mehrere Unternehmen aus dem Markt ausgeschieden wären, niemals gegründet worden wären oder fusioniert hätten. So lange keine begründeten Hinweise vorliegen, dass sich ohne das Kartell eine andere Marktstruktur entwickelt hätte, sollte aus Gründen der Praktikabilität diese Annahme getroffen werden. Bei der Ermittlung des Referenzpreises sind verschiedene Verfahren aus theoretischer Sicht denkbar und praktikabel. Beispielsweise kann der Referenzpreis kostenbasiert ermittelt werden, oder es wird ein Vergleichsmarkt herangezogen. Als Vergleichsmarkt kann auch der gleiche Markt angesehen werden entweder bevor oder nachdem ein Kartell aufgedeckt wurde. ${ }^{9}$ Welches Verfahren nun besser geeignet ist, hängt von vielen Faktoren ab, darunter wie die Preissetzung in diesem Markt erfolgt.

Mit Hilfe des Referenzpreises lässt sich der Verteilungseffekt abschätzen. Allein dies ist schon schwierig, weil es kein gesichertes Verfahren gibt, das den Referenzpreis festlegt. Um ein Vielfaches schwieriger ist es den Allokationseffekt

${ }^{9}$ Ein solcher Ansatz gibt eine verzerrte Schätzung des Preisaufschlages wieder. Diese Verzerrung müsste gesondert berücksichtigt werden. Siehe Harrington (2004). 
abzuschätzen, denn man müsste ermitteln, wie viel die Kunden zusätzlich nachgefragt hätten, wenn sie zum Referenzpreis hätten kaufen können. Diese Nachfragemenge wird beeinflusst durch die Qualität und die Preise der anderen Kartellmitglieder und auch durch die Nachfragesituation des Geschädigten. Selbst die Wahl der Produktionstechnologie kann durch den überhöhten Preis des Vorproduktes beeinflusst worden sein. Hellwig (2006) zeigt, dass der Allokationseffekt, also der entgangene Gewinn, wenn ein Unternehmen weniger produziert und verkauft, weil es überhöhte Vorleistungspreise bezahlen muss, den Verteilungseffekt überwiegt. Dennoch wird dieser Effekt bei der Ermittlung häufig vernachlässigt. $^{10}$

Bei der Diskussion um die Höhe des Schadens wird oft vorgebracht, dass der ummittelbar Geschädigte, der direkt von einem Kartellmitglied gekauft hat, den überhöhten Preis an seine Kunden wiederum weitergibt. Die passing-on defense bezeichnet dieses Argument, mit dem ein Kartellmitglied den Schadensersatzanspruch vermindern kann. Es ist zweifelsfrei richtig, dass der überhöhte Preis zumindest teilweise weitergegeben wird, so dass der Verteilungseffekt nicht in der vollen Höhe zu Lasten des unmittelbaren Käufers geht. Hellwig (2006) bemerkt, dass die Diskussion um die passing-on defense so lange unberechtigt ist, als der entgangene Gewinn aus dem Allokationseffekt außer Acht bleibt; der letztere Effekt dominiert den Verteilungseffekt und wenn er nicht berücksichtigt wird, wäre es ungerechtfertigt auch noch den entgangenen Gewinn zu kürzen.

Die passing-on defense nicht zuzulassen, sehen wir nur als Notbehelf zum Schutz der unmittelbar vom Kartell Betroffenen. Zwar ist nach Hellwig der entgangene Gewinn mindestens so hoch ist wie der Verteilungseffekt, aber er wird sich in einem Verfahren von dem Geschädigten nur schwer nachweisen und noch schwerer quantifizieren lassen.

Eine ähnlich pragmatische Lösung wäre den entgangenen Gewinn zu ersetzen und - bis auf einen gelungenen Nachweis seiner tatsächlichen Höhe - diesen in Höhe des Verteilungseffektes anzusetzen. Zusätzlich erhält der Geschädigte Schadensersatz für den Verteilungseffekt, auf dem die passing-on defense anwendbar ist. Dieser Vorschlag hat den Vorteil, dass er eine bessere Näherung des tatsächlichen Schadens darstellt als den Allokationseffekt zu ignorieren. Unberührt bleiben in jedem Fall die Ansprüche der indirekt Geschädigten. Daraus ergibt sich ein weiterer Vorteil des Vorschlages, auf den wir im nächsten Abschnitt eingehen.

Unabhängig von der passing-on defense erfahren mittelbar Betroffene, also Kunden des direkt Geschädigten, einen Schaden, der sich ebenso zusammensetzt aus dem überhöhten Preis der gekauften Einheiten und dem entgangenen Gewinn aus den wegen des überhöhten Preisen nicht gekauften Einheiten.

Ob ein mittelbarer Schaden zu einem Schadensersatzanspruch führt, wird kritisch betrachtet, doch entgegen den Entscheidungen des U.S. Supreme Courts ist

${ }^{10}$ Siehe Verboven und van Dijk (2007). 
konzeptionell ein indirekter Schaden durchaus existent. ${ }^{11}$ Daher sollen Ansprüche von indirekt Geschädigten in Europa berechtigt sein. ${ }^{12}$ In Deutschland sind die Meinungen kontrovers. ${ }^{13}$ Dass ein mittelbarer Schaden entsteht und im Sinne des Weißbuches auch zu kompensieren ist, lässt sich aus dem folgenden Gedankenexperiment herleiten:

Anstelle direkt an Kunden zu verkaufen, verkaufen die Kartellmitglieder an eine eigens gegründete Vertriebsgesellschaft, die ihre Gewinne an die Kartellmitglieder auszahlt. Die Vertriebsgesellschaft kauft die Güter zum Kartellpreis und verkauft sie zum gleichen Preis weiter.

Einen mittelbaren Schaden nicht anzuerkennen würde in diesem Falle bedeuten, dass das Kartell schadlos bleibt. Dies widerspricht im Grundsatz der im Weißbuch dargestellten Position. In realen Fällen ist es schwieriger den mittelbaren Schaden zu erkennen und zu quantifizieren. Daher ist davon auszugehen, dass mit zunehmender Entfernung vom Kartell entlang der Wertschöpfungskette auch die Kosten des Schadensnachweises derart zunehmen, dass eine Durchsetzung eines Anspruches aus ökonomischen Gründen ausgeschlossen ist.

Zusammenfassend halten wir fest, dass Schaden durch überhöhte Preise und durch verminderte Nachfrage entsteht. Die passing-on defense soll Anwendung finden und es besteht ein Anspruch auf Schadensersatz seitens der mittelbar Betroffenen. Im folgenden Abschnitt befassen wir uns mit der Anreizwirkung der passing-on defense.

\section{Passing-on defense}

Im Grundsatz hat ein Betroffener Abnehmer einen Anspruch auf Schadensersatz, der sich genau auf den tatsächlichlichen Schaden erstreckt. ${ }^{14}$ Aus diesem Grund muss ein Geschädigter das Argument der passing-on defense gegen sich gelten lassen. Die passing-on defense bezieht sich ausschließlich auf die Weitergabe von überhöhten Preisen. Der entgangene Gewinn einer nachgelagerten Wertschöpfungsstufe durch die verringerte Nachfrage bleibt von der passing-on defense unberührt. Für die Anreizwirkung des Kartellrechts ist es nicht erforderlich eine Bestrafungskomponente in die Schadensersatzzahlungen im Sinne von punitive damages einzubeziehen, denn diese Funktion wird durch das Bußgeldverfahren erfüllt. Das Bußgeld orientiert sich dann an den Umsätzen der Kartellmitglieder und nicht an den ungerechtfertigt erzielten Gewinnen.

Ein weiteres bestärkendes Argument für die passing-on defense ist, dass das Kartellmitglied nicht mehrfach in Anspruch genommen wird. Da mittelbar

\footnotetext{
${ }^{11}$ Hanover Shoe Inc. v. United Shoe Machinery Corp. 392 U.S. 481 (1968). Illinois Brick Co. et al. v. Illinois et al. 431 U.S. 720 (1977).

${ }^{12}$ Siehe Weißbuch, Europäische Kommission (2008b).

${ }^{13}$ Siehe Bundeskartellamt (2005), S.9.

${ }^{14}$ Bundeskartellamt (2005), S.9, Weißbuch, Europäische Kommission (2008b), S.10.
} 
Betroffene auch einen Anspruch auf Entschädigung haben, würde ein Kartellmitglied mehr als den tatsächlichen Schaden als Entschädigung bezahlen müssen. Dann entstünde ein exzessiver Anreiz Schadensersatz geltend zu machen und es könnten unberechtigte Klagen provoziert werden. ${ }^{15}$ Außerdem wäre es im Extremfall denkbar, dass die Gesamthöhe der Schadensersatzzahlungen über alle Grenzen wächst, wenn die Wertschöpfungskette sehr lang ist.

Praktikabilitätsgründe alleine sollten kein Argument gegen die passing-on defense sein. Genannt werden eine hohe Zahl von Anspruchsstellern und eine komplexe Beweisführung. ${ }^{16}$ Dem Prinzip des Schadensausgleichs darf nicht entgegenstehen, dass hohe Kosten der Rechtsdurchsetzung erwartet werden. Dies trifft sicherlich nicht für alle Fälle zu und hätte zur Folge, dass Schadensersatz auch in Fällen, die klar nachzuweisen sind, unterbleibt.

In die Diskussion um die passing-on defense sind auch mittelbar Betroffene einzubeziehen. Unabhängig davon welches Rechtsprinzip zur Reichweite des verletzten Schutzgesetzes zugrunde gelegt wird, ${ }^{17}$ kann maximal der gesamte ökonomische Schaden an die Geschädigten ausgezahlt werden. Im Idealfall kann jeder Geschädigte seinen Schaden genau quantifizieren und nachweisen. Es ist jedoch davon auszugehen, dass dies schwierig und mit hohen Kosten verbunden ist, so dass jeder Schaden um so schwerer nachzuweisen ist, je weiter man sich in der Wertschöpfungskette von der kartellierten Stufe entfernt, so dass entferntere Stufen auf die Durchsetzung ihres Schadens eher verzichten werden.

Welche Anreize schafft nun die Einführung der passing-on defense? Auf den ersten Blick wird ein Kartellmitglied stets versuchen mit diesem Einwand den zu zahlenden Schadensersatz zu reduzieren. Es wird also nachzuweisen versuchen, dass sein Kunde den überhöhten Einkaufspreis wiederum an seine Kunden weiter gegeben hat. Der Geschädigte wird wiederum nachzuweisen versuchen, dass sein Verkaufspreis auch bei geringeren Wettbewerbspreisen auf dem vorgelagerten kartellierten Markt nicht niedriger gewesen wäre. Ein typisches Argument wird sein, dass eine Weiterwälzung aufgrund des starken Wettbewerbs nicht möglich wäre, weil sonst die Nachfrage zurückginge. Faktisch geht es in einem solchen Streit um den Anteil des Schadens, den die geschädigte Partei wiederum an seinen Kunden weitergegeben hat.

Unter bestimmten Annahmen hat ein Kartellmitglied keinen Anreiz Kosten für die Formulierung der passing-on defense aufzuwenden, denn die alleinige Wirkung wäre, dass der Schadensersatz nicht an den unmittelbar Geschädigten, sondern an die nächste Stufe in der Wertschöpfungskette ausgezahlt wird. Wenn man anerkennt, dass auch ein mittelbar Betroffener einen Anspruch auf Schadensersatz hat, sieht man auf den zweiten Blick, dass die passing-on defense die Funktion hat, den gesamten Schadensersatz auf verschiedene Stufen zu verteilen. Gelingt es dem Kartellmitglied dem unmittelbar Geschädigten nachzuweisen, dass er den

\footnotetext{
${ }^{15}$ Siehe Rubinfeld (2009), S.900.

${ }^{16}$ Bundeskartellamt (2005), S.9.

${ }^{17}$ Siehe Bundeskartellamt (2005), S.9.
} 
überhöhten Preis zumindest teilweise abgewälzt hat, so ist dies unmittelbar der Schaden, den die zweite Stufe getragen hat. Im Idealfall kompensieren die Kartellmitglieder den gesamten Schaden, den sie verursacht haben. Dies entspricht in vollem Maße dem Anspruch des Weißbuchs. Eine wesentliche Annahme für das Zustandekommen dieses Falles ist, dass alle Parteien auf allen Stufen ihren Schaden kennen und nachweisen können und die Transaktionskosten so niedrig sind, dass sie kein Hemmnis für die Durchsetzung von Schadensersatzansprüchen darstellen. Ein konsequenter Vorschlag von Rüggeberg und Schinkel (2006) ist, dass der Gesamtschaden von einer zentralen Stelle, eine nationale Wettbewerbsbehörde oder DG COMP, ermittelt, konsolidiert und auf die verschiedenen Wirtschaftsstufen verteilt wird. Kritisch an einer solchen Vorgehensweise ist, dass die Ermittlung der Schadenshöhe und die Zuordnung auf einzelne Schädiger wegen hoher Informationskosten oder gar -defizite in vielen Fällen unmöglich sind. Dennoch würde man eine Abschätzung vornehmen müssen und dazu Recherchen durchführen. Dies ist natürlich mit Kosten verbunden, die von dieser zentralen Stelle getragen werden müssten. Während ein Kläger stets abwägt, ob der erwartete Nutzen von zusätzlichen Recherchen ihre Kosten wert sind, wäre es nahe liegend, dass man von einer zentralen Institution mehr Informationen verlangen würde, wenn man die Kosten ihrer Beschaffung nicht tragen braucht. Insofern würden die Kosten der privaten Rechtsdurchsetzung in ihrer Funktion als Maß für eine effiziente Rechtsdurchsetzung ausgehebelt, wenn sie von einer Behörde getragen würden. Es wäre exzessive Rechtsdurchsetzung zu erwarten. Vielmehr ist das Instrument der Class Action angebracht, weil es zumindest im Aggregat der Interessensvertretung Kosten und Nutzen in einer Hand vereint. Der Modus, nach dem ein Unternehmen sich an Sammelklagen beteiligen eröffnet vielfältige Gestaltungsmöglichkeiten, insbesondere, ob ein aktiver Beitritt zu einer Gemeinschaft vonnöten sein soll, oder ob ohne sein Zutun ein Unternehmen vertreten wird. ${ }^{18}$ Wir kommen nun auf die Informationsannahme zurück und diskutieren welche Auswirkungen es hat, wenn sie - wie es in der Realität anzunehmen ist - nicht erfüllt ist.

Die Kosten des Schadensnachweises bilden eine natürliche Grenze innerhalb der Wertschöpfungskette, bis zu der ein Schadensersatzanspruch durchgesetzt werden kann. Unternehmen müssen abwägen, ob die Höhe des erwarteten Schadensersatzes die Kosten der Rechtsdurchsetzung rechtfertigen. Dabei hängt die Höhe des Schadensersatzes auch davon ab, ob die Kartellmitglieder die passing-on defense vorbringen. Auch darüber müssen die Betroffenen Unternehmen Erwartungen bilden. Tatsächlich wird es mit jeder Stufe schwieriger eine Kausalität nachzuweisen und eine Quantifizierung vorzunehmen, denn Informationsprobleme sind der Beweisführung immanent.

Mit jeder weiteren Stufe in der Wertschöpfungskette wird die Kausalität des Wettbewerbsverstoßes für überhöhte Preise für Vorleistungen verwässert. Wenn die Wertschöpfungskette ausreichend lang ist, können Kartellmitglieder davon ausgehen, dass der Schaden so weit diffundiert, dass einzelne Unternehmen keinen

${ }^{18}$ Siehe hierzu Van den Bergh und Keske (2009). 
ökonomischen Anreiz mehr haben, ihren Schaden durchzusetzen. Die passing-on defense gibt den Kartellmitgliedern ein Instrument an die Hand, das es für nachgelagerte Wertschöpfungsstufen weniger attraktiv macht ihren Ersatzanspruch durchzusetzen.

Dem steht allerdings entgegen, dass ein Kartellmitglied, das die passing-on defense vorbringt, quantifizieren muss, welchen Schaden eine Stufe an die nächste weitergegeben hat. ${ }^{19}$ Damit wären schon die Informationen aufgedeckt, die Unternehmen auf der folgende Stufe vortragen müssen, um Schadensersatz hinsichtlich des Verteilungseffektes $\mathrm{zu}$ fordern. Wenn diese Informationen verwendet werden dürfen und dadurch alle Informationsprobleme des Verteilungseffektes gelöst würden, würde die passing-on defense wirkungslos. Da die se Annahmen niemals in dieser Reinform erfüllt sind, wird der angesprochene Effekt auch nur in abgeschwächter Form auftreten können. Dennoch möchten wir dies anhand eines kurzen stilisierten Beispiels demonstrieren.

Es besteht ein Potential für Absprachen zwischen Schädiger und mittelbar Betroffenem zu Lasten des unmittelbar betroffenen Klägers auf Schadensersatz. Dies liegt daran, dass auf drei Wertschöpfungsstufen über die Höhe und die Verteilung des Schadensersatzes verhandelt wird. Es sei ein Kartellmitglied K den Schadensersatzansprüchen genau eines unmittelbar betroffenen Unternehmens $U$ und genau einer mittelbar betroffenen Partei M ausgesetzt ist.

Schadensersatz besteht aus zwei Komponenten: überhöhte Preise und entgangener Gewinn durch reduzierte Nachfrage. Um die passing-on defense anzuwenden, kann $\mathrm{K}$ auf Informationen von $\mathrm{M}$ zurückgreifen. $\mathrm{K}$ und $\mathrm{M}$ können die Berechnung des entstandenen Schadens so darstellen, dass der Schaden zwar gering ist, aber vollständig von U weitergegeben wurde. Dass der Schadensersatz gering ausfällt, liegt im Interesse von $\mathrm{K}$ und dass die überhöhten Preise vollständig weiter gegeben wurden, liegt im Interesse von $\mathrm{M}$. Um darzulegen, dass die Komponente des entgangenen Gewinns gering ausfällt, muss $M$ erklären, dass es seine Nachfrage nicht zurückgefahren hat, obwohl der Preis überhöht war. Durch eine Absprache zwischen $\mathrm{K}$ und $\mathrm{M}$ können sie die von $\mathrm{K}$ zu leistende gesamte Schadensersatzzahlung reduzieren und $M$ würde einen höheren Betrag erhalten als U erklären würde, weitergereicht zu haben.

Um die passing-on defense vorzubringen, ist der Zugriff auf Preis-, Kosten- und Nachfragedaten erforderlich. Nicht alle diese Daten liegen im Zugriffsbereich des beklagten Kartellmitglieds. Vielmehr wird das beklagte Kartellmitglied diese

${ }^{19}$ Die umgekehrte Konstellation, dass der Geschädigte den Nachweis erbringen muss, welchen Teil des Kartellpreises er nicht weiterreichen konnte, wäre ungewöhnlich und umstritten. 
Dokumente vom Kläger, der zweiten Stufe, einfordern müssen oder mit den mittelbar Geschädigten auf der dritten Stufe kooperieren müssen. Hier ergibt sich ein Verhandlungs- und Kooperationspotenzial, das möglicherweise zu Lasten der zweiten Stufe geht: Der Beitrag, den Geschädigte auf der dritten Stufe zum Nachweis der passing-on defense leisten, vermag die Summe zu erhöhen, die sie selbst als Schadensersatz erhalten können und die den Schadensersatz an die zweite Stufe mindert. Im Extremfall ist der Schaden der zweiten Stufe nur ein durchlaufender Posten, wenn sie die Preiserhöhung vollständig weiter gibt. Dann hätte die zweite Stufe gar keinen Schaden erfahren.

Dass dem Kläger kein Schaden entsteht, ist nur in dem Extremfall möglich, dass kein Allokationseffekt eintritt und die gesamte Preiserhöhung an die nachfolgende Stufe weitergegeben wird. Im Normalfall ist aber die Nachfrage preiselastisch. Dies bedeutet, dass die Kunden weniger von einem Gut nachfragen, wenn sein Preis steigt. Daher würde der Kläger nicht in vollem Umfang den überhöhten Preis an seine Kunden weiter geben.

Hinsichtlich der Beweislast befinden sich Kläger und Beklagter insofern in der symmetrischen Situation als sie der jeweils anderen Partei nachweisen müssen, wie hoch der Preis, den sie von ihren Kunden verlangen, ohne das Kartell gewesen wäre. Dabei sollen die Beweisanforderungen für die passing-on defense nicht niedriger sein als das dem geschädigten Kläger hinsichtlich des Schadens obliegende Beweismaß. So wird keine der Parteien systematisch bevorzugt oder benachteiligt. Da allerdings der weiter gereichte Schaden demjenigen entspricht, dem die nachgelagerte Stufe entspricht, liegen mit einem Schadensersatzverfahren Daten bereit, die es dem mittelbar Betroffenen erleichtern seine Forderung durchzusetzen. Dies muss insofern eingeschränkt werden, als Geschäftsgeheimnisse in einem Urteil in der Regel nicht veröffentlicht werden und nach einem Vergleich eine Veröffentlichung unterbleibt.

Das extreme Ergebnis des Beispiels, dass der Schaden des unmittelbar Geschädigten ein durchlaufender Posten ist, ist nur möglich, wenn die nachfolgenden Stufen perfekt informiert sind und ihren Schaden vollständig geltend machen können. Dies ist aufgrund von Informationskosten und Kosten der Rechtsdurchsetzung nicht realistisch. Daher ist davon auszugehen, dass nicht die gesamte Wertschöpfungskette sich an Schadensersatzklagen beteiligen wird. Dies bedeutet, dass das beklagte Kartellmitglied an einer Stufe der Wertschöpfungskette die passing-on defense vorbringen können wird, die folgende Stufe aber diesen Schaden nicht mehr geltend macht. Daraus ergibt sich für ein Kartellmitglied entgegen des Beispiels doch ein Anreiz die passing-on defense anzuwenden. Die Strategie ist also die Informationssituation eines Geschädigten und dessen Rechtsdurchsetzungskosten auszunutzen, um nicht den vollen Schaden ersetzen zu müssen.

Wir halten fest, dass die passing-on defense ein notwendiges Instrument zur Realisierung der Vorgaben des Weißbuchs ist. Nur mit der passing-on defense ist es möglich, dass ein Schaden in seiner tatsächlichen Höhe kompensiert wird. Unter besonderen Umständen kann es allerdings geschehen, dass die Anwendung der 
passing-on defense zu einer Benachteiligung des Anspruchsstellers führt. Im folgenden Abschnitt befassen wir uns mit der Anreizwirkung der privaten Kartellrechtsdurchsetzung auf bestehende Kartelle.

\section{Effizienz der Kronzeugenregelung}

Die Kommission widmet ein Kapitel ihres Working Papers dem Zusammenhang zwischen privater Kartellrechtsdurchsetzung und der Wirksamkeit der Kronzeugenregelung bei Kartellen. ${ }^{20}$ Durch die Begründung des Anspruchs eines Geschädigten gegen den Kronzeugen entsteht ein Konflikt zwischen der Anreizwirkung der Straffreiheit des Kronzeugen und der Entschädigungsleistung gegenüber den Geschädigten. $^{21}$

Mehrere Vorschläge wurden in den Konsultationen des Grünbuchs vorgebracht. Das Spektrum reicht von vollständiger Freistellung von Ansprüchen durch Geschädigte des Kartells bis hin zur gesamtschuldnerischen Haftung für den gesamten vom Kartell verursachten Schaden. Je höher die mögliche Haftung des Kartellmitglieds desto geringer ist der Anreiz, die Kronzeugenregelung in Anspruch zu nehmen. Über den individuellen Anreiz hinaus, bedeutet dies auch, dass ein Kartellmitglied in geringerem Maße damit rechnen muss, dass ein anderes Kartellmitglied die Kronzeugenregelung in Anspruch nimmt. Vielmehr kann das Kartell mit überhöhten Gewinnen fortgeführt werden. Es wird also ersichtlich, dass die Ausgestaltung der Schadenshaftung durch die Kartellmitglieder und insbesondere die eines Kronzeugen erheblichen Einfluss auf die Stabilität eines Kartells haben kann. Dies muss abgewogen werden gegen den Anspruch eines Geschädigten auf vollständige Kompensation.

Indem ein Unternehmen als Kronzeuge auftritt und straffrei bleibt, hat es einerseits mehr Informationen offen gelegt als die anderen Kartellmitglieder und es hat wegen der Straffreiheit einen größeren finanziellen Spielraum. Kartellmitglieder können im Laufe der Zeit aus dem Markt ausgeschieden sein, oder aufgrund des Bußgeldes in finanzielle Engpässe gedrängt werden. Diese zwei Gründe machen den Kronzeugen besonders attraktiv für Unternehmen, die Schadensersatzansprüche geltend machen. Es ist denkbar, dass ein Kronzeuge bei der Frage der Entschädigung schlechter gestellt ist als andere Kartellmitglieder. Wenn dies der Fall ist, sollte über die Ausgestaltung der privaten Durchsetzung und der Haftungsregelung dieser Zustand abgeändert werden. ${ }^{22}$

In welchem Maße ein Kronzeuge von der vollen gesamtschuldnerischen Haftung befreit werden soll, hat uneinheitliche Reaktionen hervorgerufen. Jede Einschränkung des Haftungsumfangs des Kronzeugen würde die Haftung der übrigen Kartellmitglieder erhöhen, was als unangebracht angesehen wird. Immerhin

\footnotetext{
${ }^{20}$ Europäische Kommission (2008a), S.81ff.

${ }^{21}$ Siehe Bulst (2008), und Haucap (2009).

${ }^{22}$ Siehe Bulst (2008), S.88.
} 
scheint Einigung darüber $\mathrm{zu}$ herrschen, dass die Inanspruchnahme der Kronzeugenregelung niemandem zum Nachteil gereichen soll, insbesondere, weil dieses Unternehmen Dokumente offen gelegt hat, mit deren Hilfe sich ein Schaden leichter nachweisen und quantifizieren lässt als bei anderen Kartellmitgliedern. ${ }^{23}$ Zusätzlich soll der Kronzeuge nur denjenigen Geschädigten verpflichtet sein, die direkt oder indirekt von diesem Unternehmen ein Produkt oder eine Dienstleistung gekauft bzw. in Anspruch genommen haben.

Dieser Vorschlag kann als Kompromiss mehrere der geforderten Kriterien über die Ausgestaltung der Haftungsbegrenzung erfüllen: Erstens verlieren die Geschädigten eines Kartells nicht ihren Anspruch auf Entschädigung. Zweitens wird der Kronzeuge nicht der Gefahr ausgesetzt den gesamten Schaden aus dem Kartell zu tragen, oder ihn zumindest nach Befriedigung der Geschädigten von den anderen Kartellmitgliedern anteilsmäßig wieder einfordern zu müssen. Drittens wird den Kartellmitgliedern keine größere Last aufgebürdet als der Schaden, den sie selbst verursacht haben. Diesem Vorschlag scheint die Vorstellung zu Grunde zu liegen, dass Schäden ausschließlich durch überhöhte Preise entstehen und von dem Unternehmen verursacht wird, von dem das Produkt oder die Dienstleistung bezogen wird.

Aus ökonomischer Sicht ist die Zuordnung des Schadens zu einem bestimmten Kartellmitglied aber schwierig. Warum? Der Schaden bemisst sich nicht nur aus dem Kartellaufschlag auf den Preis, den man in einem wettbewerblichen Markt bezahlt hätte. Aufgrund des Kartellpreisgefüges oder anderer im Rahmen der Kartellabsprache festgelegter Wettbewerbsparameter kauft man vielleicht von einem anderen Anbieter als man in einem wettbewerblichen Markt gekauft hätte. Die Kaufentscheidung wird nicht nur vom Preis, sondern auch von den Produkteigeschaften und vom Service und der geographischen Lage des Verkäufers beeinflusst und bei diesen nicht-monetären Charakteristika kann noch weniger abgeschätzt werden, wie sie sich außerhalb eines Kartells entwickelt hätten. Daher kann ein Schaden (Mehrpreis) nicht eindeutig dem Verkäufer und damit einem bestimmten Kartellmitglied zugeordnet werden, sondern es ist kausal die Kartellabsprache. Damit sind alle an der Absprache beteiligten Unternehmen in Anspruch zu nehmen.

Alle Kartellbeteiligten schulden gesamtschuldnerisch. Im Weißbuch wird dies diskutiert und die Beiträge aus der Anhörung sind da nicht eindeutig. Teilweise wird auch mit dem Gerechtigkeitsargument begründet, warum die Haftung des Kronzeugen begrenzt werden sollte. Zumindest sind sich alle einig, dass der Kronzeuge nicht schlecher gestellt werden soll als andere Kartellbeteiligte. Dies umfasst auch seine Position gegenüber den Geschädigten hinsichtlich der Beweisführung, weil der Kronzeuge zumindest schon Dokumente vorgelegt hat, die auch dem Gericht oder der EU-Kommission vorliegen und aus denen hervorgeht, wie die Kartellabsprache aussah und aufrechterhalten wurde. Dieses Beweismaterial kann auch Angaben dazu erhalten, wie überhöhte Preise festgesetzt wurden. Daher

${ }^{23}$ Siehe Rubinfeld (2009), S.897. 
muss eine Regelung gefunden werden, die zwei Kriterien erfüllt: Der Kronzeuge wird nicht schlechter gestellt als die anderen Kartellmitglieder. Die Geschädigten werden nicht benachteiligt, indem Dokumente durch den Kronzeugen durch die Vorlage bei der Kommission nicht gegen den Zugriff durch Geschädigte immunisiert werden.

Eine Begrenzung der Schadensersatzverpflichtung des Kronzeugen auf die Ansprüche derjenigen Betroffenen, mit denen er eine Vertragsbeziehung hat, würde zumindest nicht systematisch den Anteil des Kronzeugen am Gesamtschaden unteroder überschätzen. ${ }^{24}$ Zusätzlich erhöht eine solche Beschränkung die Planungssicherheit für den Kronzeugen, so dass die Attraktivität der Kronzeugenregelung nicht unnötig eingeschränkt wird. Schließlich leistet eine solche Regelung auch einen Beitrag dazu, ein bestehendes Kartell $\mathrm{zu}$ destabilisieren. Ein Kartellmitglied, dass seinen Anteil am Kartellgewinn als zu gering ansieht, kann das Kartell beenden ohne sich der Gefahr übermäßiger Schadensersatzansprüchen auszusetzen.

\section{Zusammenfassung und Schlussbetrachtung}

Wir halten fest, dass die private Durchsetzung von Schadensersatzansprüchen einen Beitrag leistet, um Kartelle zu verhindern und die Schäden aufgedeckter Kartelle zu kompensieren. Die präventive Wirkung ergänzt die bisherigen Instrumente zur Aufdeckung von Kartellen. Daher werden Hardcore-Kartelle, die relativ leicht von potentiell Geschädigten aufgedeckt werden können, mit geringerer Wahrscheinlichkeit überhaupt erst eingerichtet.

Nach Aufdecken eines Kartells sollen Betroffene ihren Schaden in vollem Umfang, also die Verteilungswirkung und den Allokationseffekt, geltend machen dürfen. Kartellmitglieder sollen die passing-on defense entgegenhalten dürfen. Nur so kann prinzipiell eine Kompensation des vollen Schadens erfolgen. Dies wird nur gehemmt durch Kosten der Rechtsdurchsetzung. Die Gefahr der missbräuchlichen Kollusion zwischen Schädiger und mittelbar Geschädigten bei der Anwendung der passing-on defense besteht nur in besonderen Einzelfällen.

Dem Effekt, dass Kartelle weniger wahrscheinlich werden, steht entgegen, dass die Kronzeugenregelung weniger attraktiv wird, wenn der Kronzeuge sich mit dieser Rolle zugleich selbst Schadensersatzansprüchen aussetzt. Dies kann durch die Ausgestaltung seines Haftungsumfanges begrenzt werden und bedarf weiterer Klärung. Die Attraktivität der Kronzeugenregelung sinkt daher nicht per se. Die Zuordnung des Gesamtschadens auf die einzelnen Kartellmitglieder lässt sich konzeptionell nicht durchführen. Insbesondere ist die Zuordnung des Schadens eines betroffenen Unternehmens zu dem Kartellmitglied, mit dem es in einer

\footnotetext{
${ }^{24}$ Unbenommen bleibt eine Aufhebung dieser Beschränkung, falls sich die anderen Kartellanten später als zahlungsunfähig erweisen.
} 
Vertragsbeziehung steht, inkorrekt. Ursächlich ist alleine die Kartellvereinbarung, an der alle Kartellmitglieder beteiligt sind.

Wir befürworten die Initiative der Kommission, einen Rechtsrahmen für die private Durchsetzung des Kartellrechts zu schaffen. Bestehende methodische Ansätze greifen den weniger komplexen überhöhten Preisaufschlag auf. Dies ist allerdings nur ein Bestandteil des Schadens, da eine zurückgegangene Nachfrage einen erheblichen Bestandteil des Kartellschadens ausmachen kann. Für diese zweite Komponente sind bisher noch wenige Methoden erarbeitet und noch nicht in der Praxis anerkannt. Die Etablierung eines Rechtsrahmens für die Durchsetzung von Schadensersatzforderungen wird den Bedarf an solchen Methoden wecken und in der Folge werden sie auch entwickelt werden, denn erst jetzt entsteht hierfür die Notwendigkeit.

Es wird interessant sein, diese Entwicklung aus wissenschaftlicher Sicht zu verfolgen und zu beobachten, wie sich eine Systematik zur Durchsetzung verschiedener Arten von Kartellschäden durchsetzt. Dies wird eine durchgehende Zusammenarbeit von Ökonomen und Juristen erfordern. 


\section{Literatur}

Bulst, F.W., 2008, Of Arms and Armour - The European Commission's White Paper on Damages Actions for Breach of EC Antritrust Law, in: Bucerious Law Journal, Heft 2, 81-95.

Bundeskartellamt, 2005, Private Kartellrechtsdurchsetzung - Stand, Probleme, Perspektiven, Diskussionspapier für die Sitzung des Arbeitskreises Kartellrecht am 26. September 2005, http://cms.bundeskartellamt.de/wDeutsch/download/pdf/Diskussionsbeitraege/05_Proftag.pdf

Europäische Kommission, 2008a, Commission Staff Working Paper accompanying the White Paper on Damages actions for breach of the EC antitrust rules, COM(2008) 165 final.

Europäische Kommission, 2008b, Weißbuch Schadensersatzklagen wegen Verletzung des EG-Wettbewerbsrechts, $\operatorname{KOM(2008)~} 165$ endgültig.

Harrington, J., 2004, Post-Cartel Pricing During Litigation, in: Journal of Industrial Economics 52, 4, 517-533.

Haucap, J., 2009, Kartellrecht - Verschärfung durch Sammelklagen, in: Wirtschaftsdienst 89, 5, 286.

Hellwig, M., 2006, Private Damage Claims and the Passing-On Defense in Horizontal Price-Fixing Cases: an Economist's Perspective, Preprints of the Max Planc Institute on Collective Goods, 2006/22.

Renda, A. et al., 2007, Making antitrust damages actions more effective in the EU: welfare impact and potential scenarios, Final Report for the European Commission, DG COMP/2006/A3/012.

Rubinfeld, D.L., 2009, Evaluating Antitrust Enforcement: Economic Foundations, in: Barry E. Hawk (Hrsg.), Annual Proceedings of the Fordham Competition Law Institute, Chapter 19, 457-469.

Van den Bergh, R., S. Keske, 2009, Rechtsökonomische Aspekte der Sammelklage, in: M. Casper u.a. (Hrsg.), Auf dem Weg zu einer europäischen Sammelklage?, Sellier European Law Publisher, 17-40.

Verboven, F., T. van Dijk, 2007, Cartel damage claims and the passing-on defense, mimeo. 